\title{
Spontaneous Microsaccades Reflect Shifts in Covert Attention
}

\author{
Shlomit Yuval-Greenberg, ${ }^{1}$ Elisha P. Merriam, ${ }^{2}$ and David J. Heeger ${ }^{2}$ \\ ${ }^{1}$ School of Psychological Sciences and Sagol School of Neuroscience, Tel Aviv University, Tel Aviv 6997801, Israel, and ${ }^{2}$ Department of Psychology and \\ Center for Neural Science, New York University, New York, New York 10003
}

\begin{abstract}
Microsaccade rate during fixation is modulated by the presentation of a visual stimulus. When the stimulus is an endogenous attention cue, the ensuing microsaccades tend to be directed toward the cue. This finding has been taken as evidence that microsaccades index the locus of spatial attention. But the vast majority of microsaccades that subjects make are not triggered by visual stimuli. Under natural viewing conditions, spontaneous microsaccades occur frequently $(2-3 \mathrm{~Hz})$, even in the absence of a stimulus or a task. While spontaneous microsaccades may depend on low-level visual demands, such as retinal fatigue, image fading, or fixation shifts, it is unknown whether their occurrence corresponds to changes in the attentional state. We developed a protocol to measure whether spontaneous microsaccades reflect shifts in spatial attention. Human subjects fixated a cross while microsaccades were detected from streaming eye-position data. Detection of a microsaccade triggered the appearance of a peripheral ring of grating patches, which were followed by an arrow (a postcue) indicating one of them as the target. The target was either congruent or incongruent (opposite) with respect to the direction of the microsaccade (which preceded the stimulus). Subjects reported the tilt of the target (clockwise or counterclockwise relative to vertical). We found that accuracy was higher for congruent than for incongruent trials. We conclude that the direction of spontaneous microsaccades is inherently linked to shifts in spatial attention.
\end{abstract}

Key words: covert attention; eye movements; fixation; microsaccades; spatial attention; visual perception

\section{Introduction}

Attention and eye movements are tightly linked, but the nature of this link is not fully understood (Rizzolatti et al., 1987; Corbetta et al., 1998; Moore and Armstrong, 2003; Awh et al., 2006; Smith and Schenk, 2012). A series of studies investigated the relationship between spatial attention and miniature $\left(<1^{\circ}\right)$ saccades, called microsaccades, that occur frequently $(\sim 2-3 \mathrm{~Hz})$ when subjects fixate (Zuber et al., 1965; Winterson and Collewijn, 1976; Engbert, 2006; Rolfs, 2009; Martinez-Conde et al., 2013). Microsaccades are modulated by the onset of visual stimuli: their rate sharply drops for $\sim 150 \mathrm{~ms}$ after stimulus onset and then rebounds and peaks at 200-300 ms after stimulus onset before returning to baseline. Such stimulus-triggered microsaccades (measured during the rebound) have been shown to modulate with attention. When the visual stimulus is an endogenous (voluntary) attention cue, instructing the subject to shift attention, stimulus-triggered microsaccades tend to occur in the same direction as the cue (Hafed and Clark, 2002; Engbert and Kliegl, 2003; Laubrock et al., 2005, 2007, 2010; Hafed et al., 2011). When

\footnotetext{
Received Feb. 10, 2014; revised June 22, 2014; accepted Aug. 16, 2014.

Author contributions:S.Y.-G., E.P.M., and D.J.H. designed research; S.Y.-G. performed research; S.Y.-G. analyzed data; S.Y.-G., E.P.M., and D.J.H. wrote the paper.

This work was supported by NIH Grant R01-EY019693. S.Y.-G. was supported by the EU Marie Curie outgoing international fellowship and by the Weizmann Institute Advancing Women in Science award.

The authors declare no competing financial interests.

Correspondence should be addressed to Shlomit Yuval-Greenberg, Tel Aviv University, P.0. Box 39040, Tel Aviv 6997801, Israel. E-mail: shlomitgr@tau.ac.il.

DOI:10.1523/JNEUROSCI.0582-14.2014

Copyright $\odot 2014$ the authors $\quad 0270-6474 / 14 / 3413693-08 \$ 15.00 / 0$
}

the stimulus is an exogenous (involuntary) peripheral cue, stimulus-triggered microsaccades follow the dynamics of attention, including a shift away from the target ("inhibition of return”; Galfano et al., 2004; Rolfs et al., 2005). These findings have led some to conclude that microsaccades are an index of covert attention (Hafed and Clark, 2002; Laubrock et al., 2007, 2010).

Most studies of microsaccades and attention have focused on stimulus-triggered microsaccades. But stimulus-triggered microsaccades constitute a minority of the microsaccades that subjects make. In many experimental and naturalistic viewing conditions, microsaccades occur spontaneously during fixation and are not evoked by a stimulus or yolked to a task. Although a few studies show that spontaneous microsaccades are generated dynamically depending on visual demands (Engbert and Mergenthaler, 2006; Rucci et al., 2007; Ko et al., 2010; Poletti and Rucci, 2010), these microsaccades have been essentially ignored by studies of attention. The link between stimulus-related microsaccades and attention may not generalize to spontaneous microsaccades, which are not triggered by an attention cue, and therefore may be unrelated to attention. If microsaccades reflect the locus of attention, then it should be possible to infer the direction of attention from the direction of microsaccades occurring spontaneously during fixation, independent of any experimental manipulation.

In the current study, spontaneous microsaccades during fixation were monitored in real time. Spatial attention was not experimentally manipulated but was characterized immediately after a microsaccade by measuring performance to targets at different locations. The onset of the stimulus and the location of the target 
were contingent on the timing and the direction of microsaccades. Performance was compared for targets that were congruent (same direction) or incongruent (opposite direction) with the microsaccades. The hypothesis was that performance would be better for congruent than incongruent targets, i.e., that the direction of a spontaneous microsaccade could predict the direction of spatial attention, even for microsaccades that occurred spontaneously, i.e., when attention was not experimentally manipulated.

\section{Materials and Methods}

Subjects. Data were acquired from nine subjects. Eight subjects participated in each experiment [Experiment 1 (Exp 1), five females and three males; Exp 2, four females and four males]. Seven subjects participated in both experiments. All subjects were healthy, with no history of neurological disorders and with normal or corrected-to-normal vision. Experiments were conducted with the written consent of each subject, and the experimental protocol was approved by the University Committee on Activities Involving Human Subjects at New York University.

Stimuli. Eight grating patches (radius, $0.5^{\circ}$; spatial frequency, $3.5 \mathrm{cycle} /{ }^{\circ}$ ) were located at eight peripheral locations around fixation. The eight grating patches were arranged in a circle either surrounding the center of the screen (Exp 1) or surrounding the eye position at the time of stimulus presentation (Exp 2). In Exp 1, the patches were located at 5, 5.5, or $6^{\circ}$ eccentricity (relative to the center of the screen). The three eccentricities were presented in a randomly shuffled order, with an equal probability for each eccentricity (see Fig. $2 A$ ). In $\operatorname{Exp} 2$, the patches were located $6^{\circ}$ from the gaze position at the time of stimulus onset (see Fig. $3 A)$. Each of the gratings was independently tilted slightly either clockwise or counterclockwise (relative to vertical). The angle of the tilt was determined individually for each subject according to a pretest before the main experiments. Specifically, a tilt threshold was measured using a staircase procedure to achieve an average accuracy rate of $\sim 70 \%$. The tilts varied across subjects between 0.7 and $2.5^{\circ}\left(1.65 \pm 0.68^{\circ}\right.$, mean $\left.\pm \mathrm{SD}\right)$.

Procedure. Subjects performed an orientation discrimination task, with stimulus presentations that were contingent on microsaccades (see Fig. 2A). Subjects sat in a dark room at a distance of $57 \mathrm{~cm}$ from the display monitor and fixated a black cross $\left(0.2^{\circ}\right)$ on a gray screen. A predetection period of $2 \mathrm{~s}$ was followed by the initiation of the microsaccade detection procedure (Fig. 1A). The detection of a microsaccade offset (decrease in eye-movement velocity below threshold; see below) immediately triggered the presentation of the stimulus surrounding the fixation cross (Exp 1) or the actual eye position (Exp 2). Stimulus duration was $100 \mathrm{~ms}$. This was followed by a "postcue," a $500 \mathrm{~ms}$ presentation of a central arrow $\left(0.5^{\circ}\right)$ pointing toward one of the eight locations indicating which of them was the target. The postcue was directed either toward the grating patch located closest to the direction of the microsaccade ("congruent" trials) or toward the grating patch opposite $\left(180^{\circ}\right)$ the microsaccade ("incongruent" trials). The task was limited to only two of the eight gratings to maximize the number of congruent/incongruent trials, thereby limiting the experiment to a reasonable duration. The postcue presentation was followed by the reappearance of the black fixation cross, which stayed on until a button press was detected. The subjects' task was to indicate with one of two possible button presses whether

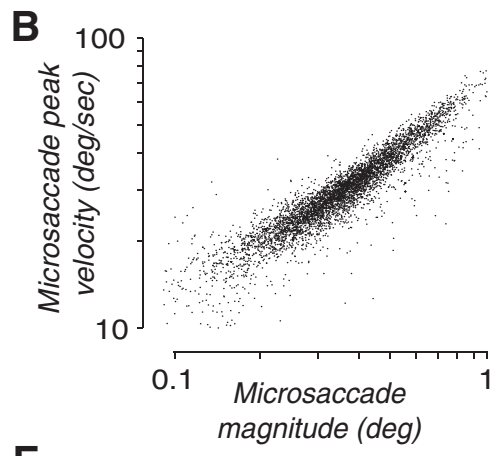

E

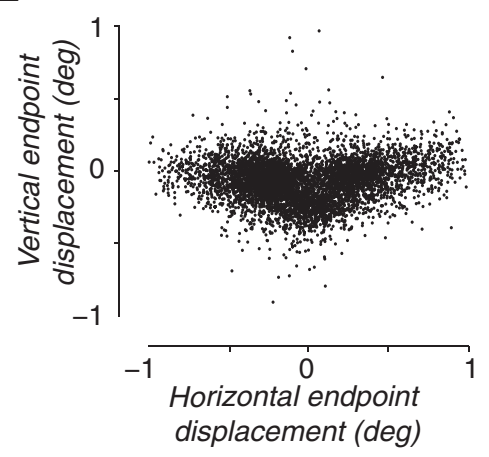

Figure 1. Real-time microsaccade detection. $\boldsymbol{A}$, Eye position during a typical trial. Black curve, Horizontal eye position; gray (directions and amplitudes), including all microsaccades of all subjects in Exp 1. Each dot represents the spatial coordinates of the endpoint of a microsaccade, normalized such that zero corresponds to the eye position just before each microsaccade.

the grating was tilted clockwise or counterclockwise of vertical. Following the button press, the fixation cross briefly $(100 \mathrm{~ms})$ changed color to green if the response was correct or to red if the response was incorrect.

In Exp 1, each subject performed 456 microsaccade-triggered trials, of which 50\% (228) were congruent and 50\% (228) incongruent. Exp 2 had fewer trials (because there was only one eccentricity): each subject performed 192 microsaccade-triggered trials, of which 96 were congruent and 96 incongruent.

Each experiment included additional untriggered trials that enabled us to measure baseline performance accuracy in the absence of microsaccades. In the untriggered trials, the time interval between the predetection period and stimulus onset was selected to be the same as that in the previous microsaccade-triggered trial. The postcue pointed randomly toward one of the eight grating patch locations. In Exp 1, each subject performed 456 untriggered trials. In Exp 2, each subject performed 192 untriggered trials.

Eye tracking. Eye movements were monitored using a remote infrared video-oculographic system (Eyelink 1000; SR Research), with a spatial resolution of $0.01^{\circ}$ and $0.25-0.5^{\circ}$ average accuracy when using a headrest. Real-time eye-gaze data were acquired using Eyelink software and MGL (http://justingardner.net/mgl) and analyzed on-line using a realtime microsaccade detection algorithm (see below) implemented in Matlab (Mathworks). The streaming data were filtered on-line using Eyelink's software (Stampe, 1993), with the "extra filtering" option enabled. Gaze position was acquired at a sampling rate of $1000 \mathrm{~Hz}$, but the real-time analysis was performed on every other sample, resulting in a $500 \mathrm{~Hz}$ sampling rate. Using a video-based eye tracker limited the accuracy of the eye-position measurements (the spatial resolution of the eye tracker is $0.01^{\circ}$ according to the manufacturer, and calibration shifts 
A

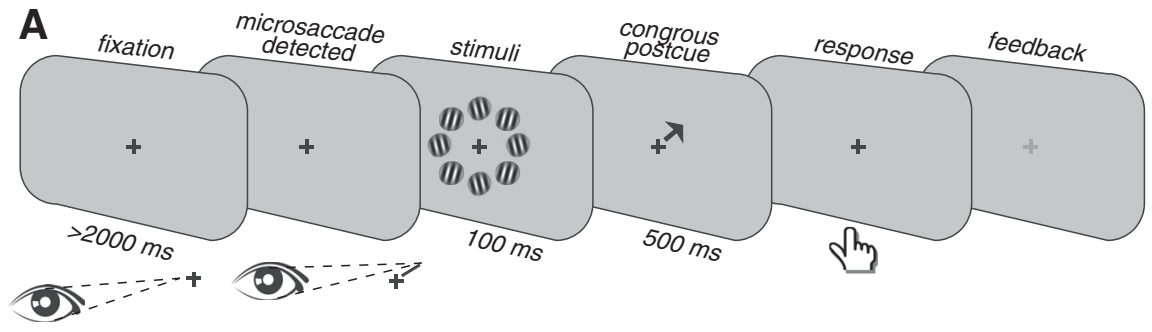

$\mathrm{B}$
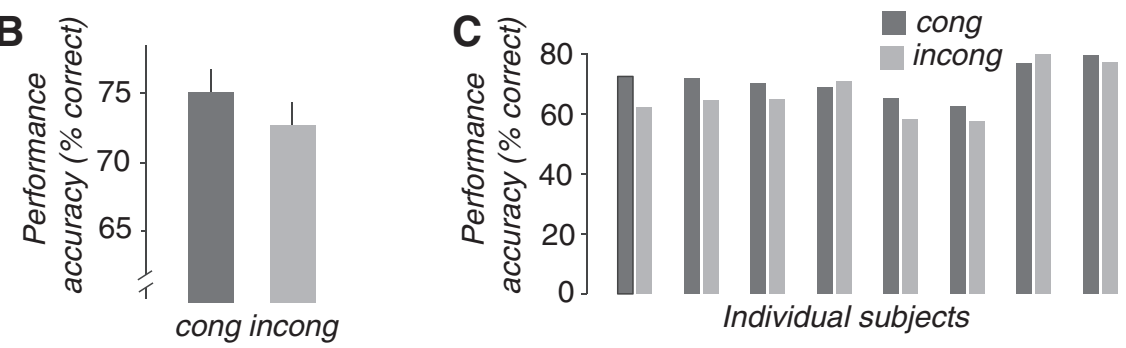

Figure 2. Experiment 1 protocol and results. $\boldsymbol{A}$, Fixation: Each trial started with $>2 \mathrm{~s}$ of fixation (duration depended on microsaccade onset time). Stimulus: After offset of the detected microsaccade, eight grating patches (shown iconically, not to scale, and not with the proper contrast) were presented for $100 \mathrm{~ms}$ at $5.5,6$, or $6.5^{\circ}$ eccentricity in a ring around fixation. Postcue: An arrow was presented for $500 \mathrm{~ms}$, pointing either in the same direction as the microsaccade (congruent) or in the opposite direction (incongruent). Response: The fixation cross reappeared, and the subject was instructed to make an orientation- discrimination decision at the cued location by pressing one of two buttons (a "1" for counterclockwise, a "2" for clockwise). Time to respond was unlimited. Feedback: The fixation cross changed color: green, correct; red, incorrect. B, Performance accuracy. Performance on congruent trials was better than on incongruent trials. C, Performance accuracy for all individual subjects. cong, Congruent; incong, incongruent.

during the experiment, adding some inaccuracies). However, these small inaccuracies of the eye tracker had no consequences for our conclusions because they were independent of the randomized order of the experiment conditions.

Microsaccades were detected in real time (Rucci et al., 2007; Chen and Hafed, 2013) using a modification of a published algorithm (Engbert and Mergenthaler, 2006). Each trial started with 2 s of eye-position recordings that were used for correcting drift in the eye-tracker calibration of the center of fixation and for setting a saccade-detection velocity threshold for that trial. When the subject blinked during this initial recording, it was repeated until a blink-free $2 \mathrm{~s}$ period of eye-position data were acquired. An elliptic threshold criterion for microsaccade detection was determined in 2D velocity space based on the horizontal and the vertical velocities of the eye-movement data measured during this $2 \mathrm{~s}$ time period. Specifically, we set the threshold to be six times the SD of the eye-movement velocity, using a median-based estimate of the SD (Engbert and Mergenthaler, 2006).

The predefined elliptic threshold criterion was then used to detect microsaccades from the streaming eye-position data. Microsaccade detection was performed on-line for a moving window of three vertical and three horizontal streaming data samples at a time, from which $2 \mathrm{D}$ velocities were calculated and compared with the elliptic threshold criterion. A microsaccade onset was detected only if three or more consecutive velocity samples were outside the ellipse. Saccade offset was defined as the first time point after a saccade for which velocity fell below the threshold. Because blinks may appear in the eye trace as saccades, followed by missing values (when the eyelid was closed), the procedure confirmed that the saccade offset was not a missing value and, therefore, that the ostensible saccade was not instead part of a blink. The process was iterated until a valid microsaccade offset was identified. It was not the case that this procedure detected each and every microsaccade, because that was not necessary. Indeed, there were certainly a large number of missed microsaccades. Rather, the procedure was designed to be conservative such that there were very few false microsaccades detected ( $<7 \%$; see next paragraph).

Eye-movement data were reanalyzed off-line after acquisition. Trials were excluded from the analysis if there were blinks within $200 \mathrm{~ms}$ (before or after) stimulus onset, or if the detected saccade was larger than $1^{\circ}$.
In addition, we visually inspected the data and excluded a few additional trials for which it was unclear whether a microsaccade had occurred. This resulted in the rejection of $0-7.1 \%$ of trials, across subjects and across the two experiments (Exp 1,3\% averaged across subjects; Exp $2,2.1 \%)$

Statistics. Nonparametric randomization tests were used to assess the statistical reliability of the differences in performance accuracy between the congruent and incongruent conditions. We shuffled the labels ("congruent" and "incongruent") 5000 times for all trials of each subject. For each random reshuffling, we averaged across subjects and recalculated the difference between conditions on the shuffled data, to obtain a null distribution for the average difference across subjects in performance accuracy. The measured average difference value was then compared with the null distribution, and the proportion of the null distribution greater than the measured value was designated as the one-tailed $p$ value. To ensure that the results did not rely mostly on one of the individual subjects, we repeated the analyses, but leaving out the subject with the largest performance difference.

Baseline performance accuracy was computed from the untriggered trials. A baseline was determined individually for each subject as a weighted average of the performance accuracies for opposite target locations, weighted by the frequencies with which microsaccades were made toward each target location. Specifically, we computed performance accuracy for targets on the right minus that for targets on the left, weighted by the frequency of microsaccades within $\pm 22.5^{\circ}$ of rightward. Likewise, for each of the other seven target locations. These baseline performance accuracies were then averaged across subjects. A randomization test was used to compare the congruity effect from the microsaccade-triggered trials with this baseline from the untriggered trials. We shuffled the target location labels (1-8) 5000 times. For each random reshuffling, we measured the baseline performance accuracy from the untriggered trials and the congruity effect (difference in performance accuracy between the congruent and incongruent conditions) from the microsaccade-triggered trials. We used these values to create a null distribution for the difference between the congruity effect and baseline. The proportion of the null distribution greater than zero was designated as the one-tailed $p$ value.

\section{Results}

\section{Experiment 1}

The real-time microsaccade detection was designed to be conservative such that there were very few false microsaccades detected ( $<7 \%$; see Materials and Methods). A few false microsaccades were detected off-line (after the data were acquired; see Materials and Methods); these trials were removed from analysis, so the psychophysical performance reported below was based on only valid microsaccades. Running the regular off-line microsaccadedetection algorithm on the same eye-movement data (Engbert and Kliegl, 2003) demonstrated the validity of the real-time microsaccade-detection algorithm. There was (by design) an increase in microsaccade rate immediately before stimulus onset (Fig. 1C). There was also a later increase in microsaccade rate triggered by subjects' button-press responses (Fig. 1, compare $C$, $D)$. The validity of the detected microsaccades was further demonstrated by showing that they followed the main sequence of saccades (Zuber et al., 1965; Bahill, 1974; Fig. 1B). 
Orientation-discrimination performance was significantly higher for congruent trials than incongruent trials (Fig. $2 B, C$; accuracy for congruent condition: $75.1 \%$, averaged across subjects; accuracy for incongruent condition: $72.6 \% ; p=0.002$, randomization test; see Materials and Methods). To ensure that the results did not rely mostly on one of the individual subjects, we repeated the analyses, but leaving out the subject with the largest performance difference. With this subject left out, orientationdiscrimination performance was again significantly higher for congruent trials than incongruent trials $(p=0.01)$.

A complementary analysis confirmed that the interpretation of these results was not confounded by a performance bias. The distribution of microsaccade directions is biased such that some directions are more frequent than others (Engbert, 2006). Also, there are well characterized differences in performance accuracy, called "performance fields," for different locations in the visual field (Carrasco et al., 2001). If the bias in microsaccade direction was correlated with the performance field, either individually for each subject or on average across subjects, then this might have artificially inflated the congruity effect. For example, assume that a particular subject made more rightward than leftward microsaccades and that his or her discrimination performance (regardless of microsaccades) was better for stimuli placed to the right than to the left of fixation. The experimental procedure chose each target location according to microsaccade direction, so more rightward (than leftward) microsaccades would have yielded more congruent trials with the target on the right (than on the left), and vice versa for incongruent trials. Because performance (for this hypothetical subject) was better for targets located on the right (than on the left), this could have resulted in a sham congruity effect.

We found no evidence for a correlation between the bias in microsaccade direction and the performance field. There was a large horizontal bias of microsaccade direction (Fig. 1E) evident in all subjects (the average number of leftward and rightward microsaccades was larger than the average number of upward and downward microsaccades, for all subjects) and a smaller upward bias (the average number of upward, up-leftward, and uprightward microsaccades was larger than the number of downward, down-leftward, and down-rightward microsaccades in six of eight subjects). Averaged across subjects, $29 \%$ of microsaccades were directed to the right, $7.5 \%$ were directed up-rightward, $4.8 \%$ upward, $1.2 \%$ up-leftward, $41.6 \%$ leftward, $2.7 \%$ down-leftward, $0.7 \%$ downward, and $1.9 \%$ down-rightward. The performance accuracy in trials that were triggered by microsaccades, averaged across subjects, was as follows: $77.4 \%$ when the cue appeared to the right of fixation; $73.3 \%$ for an up-rightward cue; $76.3 \%$ for an upward cue; $57 \%$ for an up-leftward cue; $77 \%$ for a leftward cue; $70.1 \%$ for a down-leftward cue; $61.4 \%$ for a downward cue; and $70.2 \%$ for a down-rightward cue. The correlation between the mean performance-field bias and the mean microsaccadedirection bias was not statistically significant $(r=0.46, p=0.25)$, although for certain directions in certain subjects there were almost no microsaccades and, correspondingly, not enough microsaccade-triggered trials to measure performance reliably.

To further ensure that this correlation did not create a sham congruity effect, we measured baseline performance accuracy for trials that were not triggered by microsaccades (see Materials and Methods). These untriggered trials were randomly interleaved with the microsaccade-triggered trials, and they were equal in number. Baseline performance accuracy was computed from the untriggered trials, individually for each subject, as a weighted average of the performance accuracies for opposite target locations, weighted by the frequencies with which microsaccades were made toward each target location (see Materials and Methods). Baseline performance accuracy for the untriggered trials was statistically indistinguishable from 0 (mean baseline performance accuracy, $0.05 \% ; p=0.13$, randomization test). The difference in performance (congruent - incongruent) for microsaccade-triggered trials was significantly larger than the untriggered baseline ( $p=0.03$, randomization test; see Materials and Methods).

Microsaccades constitute a shift in the position of the eye gaze, which is either toward the target (congruent) or away from the target (incongruent). Therefore, the difference in performance between the congruent and the incongruent trials might have been explained by the shorter distance between the target and the eye position in the congruent compared with the incongruent conditions. Indeed, we observed that the congruity effect was larger for larger microsaccades $\left(0.5-1^{\circ}\right)$, which created a shorter eye-to-target distance, than for smaller microsaccades $\left(0-0.5^{\circ}\right)$. This may suggest that some of the congruity effect we found can be explained by retinal eccentricity. However, such large microsaccades constituted a very small minority (3.3\%, on average) of the microsaccades on which the gaze contingent procedure was based. Therefore, most of the retinal displacements caused by microsaccades were smaller than $0.5^{\circ}$ and were unlikely to impact perception at the peripheral eccentricity of $5-6^{\circ}$. Moreover, the congruity effect was significant even when taking only a subset of small microsaccades (e.g., microsaccades that were smaller than $0.3^{\circ} ; p=0.004$ ).

To further test the involvement of small retinal displacements in the reported difference in performance between the congruent and the incongruent trials, we compared performance for a more peripheral congruent target with a more foveal incongruent target. Since most microsaccades were smaller than $0.5^{\circ}$, we compared a congruent target at $6^{\circ}$ with an incongruent target at $5.5^{\circ}$. Performance accuracy was higher for congruent targets located at $6^{\circ}$ than for incongruent targets located at $5.5^{\circ}$ (congruent, $76.7 \%$; incongruent, $68.6 \% ; p<0.001$, randomization test), supporting the conclusion that the difference between congruent and incongruent trials cannot be accounted for by any difference in retinal location. But there was no evidence for a difference in performance between incongruent targets at $5^{\circ}$ and congruent targets at either 5.5 or $6^{\circ}$ (n.s., randomization test). It was not possible, therefore, to completely rule out any contribution of retinal displacement (i.e., shorter eye-to-target distances) to the performance differences in Exp 1, and Exp 2 was designed as a complementary approach to test the possible influence of target retinal location.

\section{Experiment 2}

The second experiment was similar to the first except that the patches were located $6^{\circ}$ from the measured eye position at the time of stimulus onset, i.e., after the microsaccade (Fig. 3A). Consequently, the eccentricity of the target (distance from center of gaze) was equal for congruent and incongruent trials.

The results replicated the congruity effect of Exp 1. Performance accuracy was significantly higher for congruent than for incongruent trials (Fig. 3B, C; congruent, $71.07 \%$; incongruent, $66.96 \% ; p<0.001$, randomization test). This difference in performance remained significant after leaving out the subject with the largest effect $(p<0.001)$.

Following the same analysis as in Exp 1, we examined the relationship between performance-field and microsaccadedirection bias. There was again a large horizontal bias of microsaccade direction and a smaller upward bias. Averaged across 
A
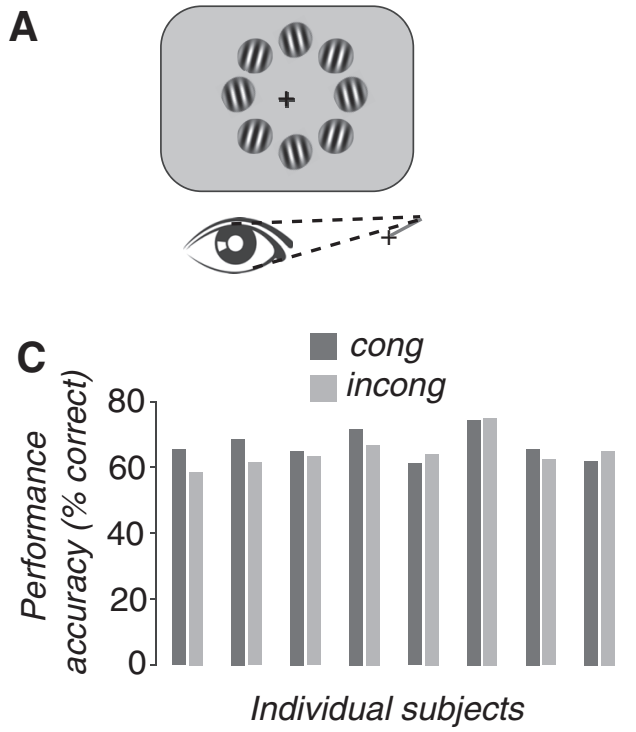

B

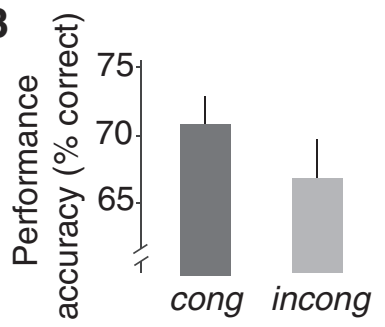

D

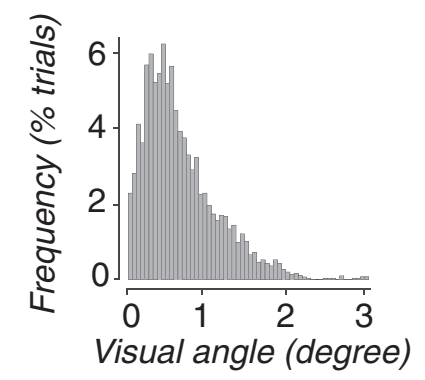

Figure 3. Experiment 2 protocol and results. $\boldsymbol{A}$, The stimulus consisted of eight grating patches (shown iconically, not to scale, and not with the proper contrast) presented for $100 \mathrm{~ms}$ at $6^{\circ}$ in a ring around the actual gaze position at the time of stimulus onset. The rest of the procedure was identical to Exp 1 (Fig. 2A). B, Performance accuracy. Performance on congruent trials was better than on incongruent trials. C, Performance accuracy for all individual subjects. $\boldsymbol{D}$, Frequency of the distances between the center of the stimulus and the fixation cross, for all subjects and trials, at the time of stimulus onset. cong, Congruent; incong, incongruent.

subjects, $27.7 \%$ of microsaccades were directed rightward, 9.4\% up-rightward, $6.6 \%$ upward, $16.2 \%$ up-leftward, $34.1 \%$ leftward, $1.5 \%$ down-leftward, $1.5 \%$ downward, and $2.9 \%$ down-rightward. The performance accuracy in trials that were triggered by microsaccades, averaged across subjects, was as follows: $71.4 \%$ when the cue appeared to the right of fixation; $69 \%$ for an up-rightward cue; $61.1 \%$ for an upward cue; $70.4 \%$ for an up-leftward cue; $71.5 \%$ for a leftward cue; $68.8 \%$ for a downleftward cue; $59 \%$ for a downward cue; and $57.8 \%$ for a downrightward cue. The correlation between the mean performance-field bias and the mean microsaccade-direction bias was statistically significant $(r=0.71, p=0.047)$. This result cannot be considered entirely meaningful because for certain directions in certain subjects, there were almost no microsaccades and, correspondingly, not enough microsaccade-triggered trials to measure performance reliably. But it does leave open the possibility that the performance difference for congruent versus incongruent trials might have been confounded with the performance-field bias, i.e., that it was a sham congruity effect.

The possibility of such a sham congruity effect was ruled out by measuring baseline performance accuracy for trials that were not triggered by microsaccades, following the same analysis as in Exp 1. Baseline performance accuracy for the untriggered trials was negative (mean baseline performance accuracy, $-3 \%$ ), and the difference in performance (congruent - incongruent) for microsaccade-triggered trials was significantly larger than the untriggered baseline ( $p=0.03$, randomization test). This means that the performance bias contributed an effect that was opposite to the congruity effect and is partially masking the "real" congruity effect. The value that we measured, which includes both the real congruity effect and the performance bias, is, in fact, smaller than the real congruity effect, and therefore the performance bias is not a concern in interpreting these data.

The grating patches were never presented nearby the fixation cross, minimizing possible concerns that the fixation cross might have inadvertently cued or masked the grating patches. The distance between the fixation cross at the center of the screen and the measured eye position at the time of stimulus onset was $0.73 \pm 0.71^{\circ}$ (mean $\pm \mathrm{SD}$ ), averaged across subjects and trials. It was $0.73 \pm 0.9,0.72 \pm 0.85$, and $0.72 \pm 0.83^{\circ}$ at 25,50 , and $75 \mathrm{~ms}$ after stimulus onset, respectively (Fig. 3D).

\section{Discussion}

When subjects fixate, microsaccades occur spontaneously and involuntarily a few times per second. These microsaccades are not necessarily triggered by a change in visual stimulation or by any other experimental manipulations. We studied the relationship between these spontaneous microsaccades and attention shifts. Immediately after a spontaneous microsaccade, performance accuracy was higher for a target located in the direction of the microsaccade (congruent) compared with targets located opposite the microsaccade direction (incongruent). We found similar results in two experiments: Exp 2 not only replicated the basic finding from Exp 1 but also complemented Exp 1 by controlling the retinal eccentricity of the targets. This finding demonstrates that spontaneously occurring microsaccades are linked to spontaneous shifts in spatial attention.

\section{Microsaccades are an index of covert attention}

Previous studies have concluded that microsaccades are "an index of attention" (Laubrock et al., 2007) or "reflect attention" (Hafed and Clark, 2002). Such studies examined stimulustriggered microsaccades that were evoked by the stimuli (including the attention cues) used in the experiment (Hafed and Clark, 2002; Engbert and Kliegl, 2003; Laubrock et al., 2005, 2007, 2010; Hafed et al., 2011). Although initially controversial (Horowitz et al., 2007), it has been established clearly and convincingly that such stimulus-triggered microsaccades modulate with attention (Laubrock et al., 2010). However, it was conceivable that spontaneous microsaccades are governed by a distinct physiological mechanism that is not influenced by attention.

In our study, we tested the hypothesis that the locus of attention and microsaccade direction are linked not only for stimulustriggered microsaccades but also for spontaneous microsaccades. This finding is an indication that some of the spontaneously occurring microsaccades are correlated with spontaneous shifts of attention and that the direction of microsaccades (on average) is an index of spatial attention. It does not, however, indicate that individual microsaccades can be used as an index of attention. It is likely that some spontaneously occurring microsaccades are linked with shifts in attention and other spontaneously occurring microsaccades are not. Our results encompass an average of the two. Hence, a single microsaccade cannot be used as an index for a specific shift of attention, but the average dynamics of microsaccades over many repetitions does reflect attention.

A theoretical framework based on Bayesian priors (weights specifying the prior probability of a target location) has been used to explain improvements in perceptual performance after the presentation of a valid attention cue (Eckstein et al., 2002, 2006, 2009; Yu and Dayan, 2005). Unlike most studies on attention, our experimental design included no precue, and attention allocation 
was based on priors and spontaneous dynamics. Subjects may have learned to expect a peripheral stimulus at specific locations, and these locations may have been asymmetrically attended as indicated by the congruity effect. These results suggest that Bayesian weights may change dynamically over time and space (even without explicit cues) and that these dynamics are linked to the direction of microsaccades.

\section{Microsaccades and visuomotor maps}

Microsaccades resemble saccades in almost every way (Zuber et al., 1965; Van Gisbergen and Robinson, 1981; Sparks, 2002; Engbert and Mergenthaler, 2006; Otero-Millan et al., 2008; Hafed et al., 2009), so they might reflect the same physiological phenomenon, located at different points on a continuum of saccade amplitudes (Hafed et al., 2009; Hafed and Krauzlis, 2012). Eye movements are controlled by neural activity in visuomotor maps in the superior colliculus (SC), frontal eye field, and lateral intraparietal area (Andersen et al., 1990; Schall et al., 1995; Corbetta et al., 1998; Hanes et al., 1998; Krauzlis, 2003; Carello and Krauzlis, 2004; McPeek and Keller, 2004; Baker et al., 2006). An eye movement occurs when there is an accumulation of activity that exceeds a threshold in a subpopulation of neurons selective for a specific direction and amplitude (Van Gisbergen and Robinson, 1981; van Opstal and van Gisbergen, 1990; Gandhi and Katnani, 2011). During fixation, neural activity in SC visuomotor maps corresponds to a stochastic process distributed around the center of the visual field, with the peak of activity from neurons coding zero amplitude and direction ("fixation-selective" neurons). Strong and continuous activity in fixation-selective neurons, relative to weaker subthreshold activity in the rest of the visuomotor map, is necessary for successful maintenance of fixation. Microsaccades may occur when the balance between fixation-selective and other neurons is momentarily tipped, causing a slight shift of the center of mass of activity away from zero (Hafed and Clark, 2002; Engbert and Kliegl, 2003; Laubrock et al., 2005, 2007, 2010; Rolfs et al., 2005, 2008; Hafed et al., 2009, 2011; Hafed and Krauzlis, 2010; Hafed and Lovejoy, 2013). This can either occur randomly, when activity in a nonfixation subpopulation randomly crosses a threshold, or it can be caused by competition between fixation-selective neurons and other subpopulations of neurons in visuomotor maps. In particular, shifts of attention can cause such momentary imbalance in the superior colliculus (Hafed and Lovejoy, 2013).

\section{Attention and visuomotor maps}

The "premotor theory" of attention (PMTA) posits that shifts of attention are based on planning goal-directed actions toward the attended location (Rizzolatti et al., 1987). One of the main predictions of this theory is that attention shifts and saccades are driven by the same neural mechanisms. Although the theory is controversial (Deubel and Schneider, 1996; Juan et al., 2004; Awh et al., 2006; Pouget et al., 2009; Gregoriou et al., 2012; Smith and Schenk, 2012), there is indeed evidence showing that shifts of spatial attention activate the same neural subpopulations that are activated by saccades to the same locations (Corbetta et al., 1998; Grosbras and Paus, 2002; Moore and Armstrong, 2003; Muggleton et al., 2003; Carello and Krauzlis, 2004; McPeek and Keller, 2004; Moore and Fallah, 2004; Müller et al., 2005; Smith et al., 2005; Awh et al., 2006). A "microsaccade variation" of the PMTA could posit that although the activity involved with attention shifts is not strong enough to evoke a large saccade to the attended location, it could be strong enough to tip the balance in visuospatial maps and trigger a microsaccade toward the attended location (Hafed and Clark, 2002; Hafed and Krauzlis, 2010). Alternatively, the opposite direction of causality could also be hypothesized, i.e., that microsaccades induce the performance enhancement we call "covert attention" (Hafed, 2013). In both cases, contrary to the traditional view of covert attention, it does involve an actual eye movement in the attended direction (Engbert and Kliegl, 2003). This hypothesis is consistent with previous findings showing that microsaccades are congruent with attention cues (Hafed and Clark, 2002; Engbert and Kliegl, 2003; Laubrock et al., 2005, 2010; Rolfs et al., 2005; Hafed et al., 2009; Hafed and Krauzlis, 2010), and also with our current finding that the direction of spontaneous microsaccades during fixation is related to shifts of attention.

Our results can be couched in the context of a computational model that purports to explain the timing and direction of microsaccades (Engbert et al., 2011; Engbert, 2012). This model is based on the notion that fixational eye movements are equivalent to a self-avoiding random walk with a $2 \mathrm{D}$ activation map that biases the random walk toward low values of activation. To stimulate the attempt to "fixate" and the influence of perceptual and attentional modulations, a quadratic function is added to the activation map, with a minimum at the fixated location. In each time step, additional activation is added to the site corresponding to the current eye position, and activation is gradually decreased at all other sites. According to the model, microsaccades occur when the walk reaches a position at which the activation surpasses a certain threshold, evoking a fast movement to the position with the global minimum of activation. The current results are compatible with this model assuming either directions of causality described above: that spontaneous fluctuations in the activation map trigger microsaccades or that microsaccades distort the activation map, which is then translated into the behavioral modulation we call spatial attention (Engbert et al., 2011).

Studies on predictive remapping of attention suggest that shifts of attention to a saccade target precedes the actual eye motion (Merriam, 2003; Cavanagh et al., 2010; Rolfs et al., 2011). However, our procedure characterized attention only after microsaccade offset. This temporal relationship between microsaccade and stimulus is inevitable in our design because one cannot anticipate when a microsaccade is going to occur. Characterizing attention concurrent with a microsaccade would require an entirely different design, including high-rate presentation of stimuli that would occasionally occur at the desired time. But doing this would miss the point of measuring spontaneous microsaccades because it would highly modulate the microsaccade rate. Although measuring attention only after microsaccade offset is a necessity of design, it does not undermine our conclusion. First, the temporal dynamics of attention shifts are substantially slower than the $\sim 70 \mathrm{~ms}$ gap that is introduced by the remapping of attention $(\sim 50 \mathrm{~ms})$ and by the actual saccade $(\sim 20 \mathrm{~ms})$. From the time of remapping to the presentation of the stimulus, there is not enough time for a second attention shift. Second, this time gap would work against our reported effect. Support for our conclusion is stronger because we find a significant effect despite the time gap.

\section{Conclusion}

Visual discrimination performance is better when the microsaccade is in the direction of the target than when it is in the opposite direction. This finding implies a relationship between the locus of spatial attention and the direction of the spontaneous microsaccades during fixation. The correlation between attention shifts and microsaccades opens the possibility of studying the dynamics 
of spontaneous shifts in attention via microsaccades. Future studies could use the procedure described here to examine the temporal dynamics of attention and to compare performance with and without microsaccades.

\section{References}

Andersen RA, Bracewell RM, Barash S, Gnadt JW, Fogassi L (1990) Eye position effects on visual, memory, and saccade-related activity in areas LIP and 7a of macaque. J Neurosci 10:1176-1196. Medline

Awh E, Armstrong KM, Moore T (2006) Visual and oculomotor selection: links, causes and implications for spatial attention. Trends Cogn Sci 10: 124-130. CrossRef Medline

Bahill A (1974) The main sequence, a tool for studying human eye movements. Math Biosci 24:191-204.

Baker JT, Patel GH, Corbetta M, Snyder LH (2006) Distribution of activity across the monkey cerebral cortical surface, thalamus and midbrain during rapid, visually guided saccades. Cereb Cortex 16:447-459. CrossRef Medline

Carello CD, Krauzlis RJ (2004) Manipulating intent-evidence for a causal role of the superior colliculus in target selection. Neuron 43:575-583. CrossRef Medline

Carrasco M, Talgar CP, Cameron EL (2001) Characterizing visual performance fields: effects of transient covert attention, spatial frequency, eccentricity, task and set size. Spat Vis 15:61-75. CrossRef Medline

Cavanagh P, Hunt AR, Afraz A, Rolfs M (2010) Visual stability based on remapping of attention pointers. Trends Cogn Sci 14:147-153. CrossRef Medline

Chen CY, Hafed ZM (2013) Postmicrosaccadic enhancement of slow eye movements. J Neurosci 33:5375-5386. CrossRef Medline

Corbetta M, Akbudak E, Conturo TE, Snyder AZ, Ollinger JM, Drury HA, Linenweber MR, Petersen SE, Raichle ME, Van Essen DC, Shulman GL (1998) A common network of functional areas for attention and eye movements. Neuron 21:761-773. CrossRef Medline

Deubel H, Schneider WX (1996) Saccade target selection and object recognition: evidence for a common attentional mechanism. Vision Res 36: 1827-1837. CrossRef Medline

Eckstein MP, Shimozaki SS, Abbey CK (2002) The footprints of visual attention in the Posner cueing paradigm revealed by classification images. J Vis 2:25-45. Medline

Eckstein MP, Drescher BA, Shimozaki SS (2006) Attentional cues in real scenes, saccadic targeting, and Bayesian priors. Psychol Sci 17:973-980. CrossRef Medline

Eckstein MP, Peterson MF, Pham BT, Droll JA (2009) Statistical decision theory to relate neurons to behavior in the study of covert visual attention. Vision Res 49:1097-1128. CrossRef Medline

Engbert R (2006) Microsaccades: a microcosm for research on oculomotor control, attention, and visual perception. Prog Brain Res 154:177-192. CrossRef Medline

Engbert R (2012) Computational modeling of collicular integration of perceptual responses and attention in microsaccades. J Neurosci 32:80358039. CrossRef Medline

Engbert R, Kliegl R (2003) Microsaccades uncover the orientation of covert attention. Vision Res 43:1035-1045. CrossRef Medline

Engbert R, Mergenthaler K (2006) Microsaccades are triggered by low retinal image slip. Proc Natl Acad Sci U S A 103:7192-7197. CrossRef Medline

Engbert R, Mergenthaler K, Sinn P, Pikovsky A (2011) An integrated model of fixational eye movements and microsaccades. Proc Natl Acad Sci U S A 108:E765-E770. CrossRef Medline

Galfano G, Betta E, Turatto M (2004) Inhibition of return in microsaccades. Exp Brain Res 159:400-404. CrossRef Medline

Gandhi NJ, Katnani HA (2011) Motor functions of the superior colliculus. Annu Rev Neurosci 34:205-231. CrossRef Medline

Gregoriou GG, Gotts SJ, Desimone R (2012) Cell-type-specific synchronization of neural activity in FEF with V4 during attention. Neuron 73:581594. CrossRef Medline

Grosbras MH, Paus T (2002) Transcranial magnetic stimulation of the human frontal eye field: effects on visual perception and attention. J Cogn Neurosci 14:1109-1120. CrossRef Medline

Hafed ZM (2013) Alteration of visual perception prior to microsaccades. Neuron 77:775-786. CrossRef Medline
Hafed ZM, Clark JJ (2002) Microsaccades as an overt measure of covert attention shifts. Vision Res 42:2533-2545. CrossRef Medline

Hafed ZM, Krauzlis RJ (2010) Microsaccadic suppression of visual bursts in the primate superior colliculus. J Neurosci 30:9542-9547. CrossRef Medline

Hafed ZM, Krauzlis RJ (2012) Similarity of superior colliculus involvement in microsaccade and saccade generation. J Neurophysiol 107:1904-1916. CrossRef Medline

Hafed ZM, Lovejoy LP (2013) Superior colliculus inactivation alters the relationship between covert visual attention and microsaccades. Eur J Neurosci 37:1169-1181. CrossRef Medline

Hafed ZM, Goffart L, Krauzlis RJ (2009) A neural mechanism for microsaccade generation in the primate superior colliculus. Science 323:940-943. CrossRef Medline

Hafed ZM, Lovejoy LP, Krauzlis RJ (2011) Modulation of microsaccades in monkey during a covert visual attention task. J Neurosci 31:15219-15230. CrossRef Medline

Hanes DP, Patterson WF 2nd, Schall JD (1998) Role of frontal eye fields in countermanding saccades: visual, movement, and fixation activity. J Neurophysiol 79:817-834. Medline

Horowitz TS, Fine EM, Fencsik DE, Yurgenson S, Wolfe JM (2007) Fixational eye movements are not an index of covert attention. Psychol Sci 18:356-363. CrossRef Medline

Juan CH, Shorter-Jacobi SM, Schall JD (2004) Dissociation of spatial attention and saccade preparation. Proc Natl Acad Sci U S A 101:15541-15544. CrossRef Medline

Ko HK, Poletti M, Rucci M (2010) Microsaccades precisely relocate gaze in a high visual acuity task. Nat Neurosci 13:1549-1553. CrossRef Medline

Krauzlis RJ (2003) Neuronal activity in the rostral superior colliculus related to the initiation of pursuit and saccadic eye movements. J Neurosci 23:4333-4344. Medline

Laubrock J, Engbert R, Kliegl R (2005) Microsaccade dynamics during covert attention. Vision Res 45:721-730. CrossRef Medline

Laubrock J, Engbert R, Rolfs M, Kliegl R (2007) Microsaccades are an index of covert attention: commentary on Horowitz, Fine, Fencsik, Yurgenson, and Wolfe (2007). Psychol Sci 18:364-366; discussion 367-368. CrossRef Medline

Laubrock J, Kliegl R, Rolfs M, Engbert R (2010) When do microsaccades follow spatial attention? Atten Percept Psychophys 72:683-694. CrossRef Medline

Martinez-Conde S, Otero-Millan J, Macknik SL (2013) The impact of microsaccades on vision: towards a unified theory of saccadic function. Nat Rev Neurosci 14:83-96. CrossRef Medline

McPeek RM, Keller EL (2004) Deficits in saccade target selection after inactivation of superior colliculus. Nat Neurosci 7:757-763. CrossRef Medline

Merriam EP, Genovese CR, Colby CL (2003) Spatial updating in human parietal cortex. Neuron 39:361-373. CrossRef Medline

Moore T, Armstrong KM (2003) Selective gating of visual signals by microstimulation of frontal cortex. Nature 421:370-373. CrossRef Medline

Moore T, Fallah M (2004) Microstimulation of the frontal eye field and its effects on covert spatial attention. J Neurophysiol 91:152-162. CrossRef Medline

Muggleton NG, Juan CH, Cowey A, Walsh V (2003) Human frontal eye fields and visual search. J Neurophysiol 89:3340-3343. CrossRef Medline

Müller JR, Philiastides MG, Newsome WT (2005) Microstimulation of the superior colliculus focuses attention without moving the eyes. Proc Natl Acad Sci U S A 102:524-529. CrossRef Medline

Otero-Millan J, Troncoso XG, Macknik SL, Serrano-Pedraza I, MartinezConde S (2008) Saccades and microsaccades during visual fixation, exploration, and search: foundations for a common saccadic generator. J Vis 8:21.1-21.18. CrossRef Medline

Poletti M, Rucci M (2010) Eye movements under various conditions of image fading. J Vis 10:6.1-6.18. CrossRef Medline

Pouget P, Stepniewska I, Crowder EA, Leslie MW, Emeric EE, Nelson MJ, Schall JD (2009) Visual and motor connectivity and the distribution of calcium-binding proteins in macaque frontal eye field: implications for saccade target selection. Front Neuroanat 3:2. CrossRef Medline

Rizzolatti G, Riggio L, Dascola I, Umiltá C (1987) Reorienting attention across the horizontal and vertical meridians: evidence in favor of a premotor theory of attention. Neuropsychologia 25:31-40. CrossRef Medline 
Rolfs M (2009) Microsaccades: small steps on a long way. Vision Res 49: 2415-2441. CrossRef Medline

Rolfs M, Engbert R, Kliegl R (2005) Crossmodal coupling of oculomotor control and spatial attention in vision and audition. Exp Brain Res 166: 427-439. CrossRef Medline

Rolfs M, Kliegl R, Engbert R (2008) Toward a model of microsaccade generation: the case of microsaccadic inhibition. J Vis 8:5.1-5.23. CrossRef Medline

Rolfs M, Jonikaitis D, Deubel H, Cavanagh P (2011) Predictive remapping of attention across eye movements. Nat Neurosci 14:252-256. CrossRef Medline

Rucci M, Iovin R, Poletti M, Santini F (2007) Miniature eye movements enhance fine spatial detail. Nature 447:852-855. CrossRef Medline

Schall JD, Hanes DP, Thompson KG, King DJ (1995) Saccade target selection in frontal eye field of macaque. 1. Visual and premovement activation. J Neurosci 15:6905-6918. Medline

Smith DT, Schenk T (2012) The premotor theory of attention: time to move on? Neuropsychologia 50:1104-1114. CrossRef Medline

Smith DT, Jackson SR, Rorden C (2005) Transcranial magnetic stimulation of the left human frontal eye fields eliminates the cost of invalid endogenous cues. Neuropsychologia 43:1288-1296. CrossRef Medline

Sparks DL (2002) The brainstem control of saccadic eye movements. Nat Rev Neurosci 3:952-964. CrossRef Medline

Stampe DM (1993) Heuristic filtering and reliable calibration methods for video-based pupil-tracking systems. Behav Res Methods Instruments Comp 25:137-142. CrossRef

Van Gisbergen JA, Robinson DA, Gielen S (1981) A quantitative analysis of generation of saccadic eye movements by burst neurons. J Neurophysiol 45:417-442. Medline

van Opstal AJ, van Gisbergen JA (1990) Role of monkey superior colliculus in saccade averaging. Exp Brain Res 79:143-149. CrossRef Medline

Winterson BJ, Collewijn H (1976) Microsaccades during finely guided visuomotor tasks. Vision Res 16:1387-1390. CrossRef Medline

Yu A, Dayan P (2005) Inference, attention, and decision in a Bayesian neural architecture. NIPS 17:1577-1584.

Zuber BL, Stark L, Cook G (1965) Microsaccades and the velocityamplitude relationship for saccadic eye movements. Science 150:14591460. CrossRef Medline 